DEMONSTRATIO MATHEMATICA

Vol. $\mathrm{XX} \quad$ No $3.4 \quad 1987$

Brian Fisher, Todor D. Todorov

\title{
OPERATIONS WITH DISTRIBUTION VECTORS
}

The space of distributions $D^{\prime}$ is isomorphicalls embedded in the space of distribution vectors $\stackrel{\sim}{\prime}^{\prime}(1)$ and this larger space $D^{\prime}$ is equipped with operations of multiplication and integration. Several formulae for $\delta^{2}(x), \delta^{(p)} . \delta^{(q)}$, $x_{+}^{p} \cdot \delta^{(g)}(x)$, etc., are derived which, as we know, are significant for some appilcations, in particular, in quantum field theory but they do not make sense in $D^{\prime}$ itself. The paper is a continuation of a previous work (1) but it could be read inde pendently.

In the following we let $C$ be the field of complex numbers, $D$ be the space of infinttely differentiable functions defined in the real line with compact support and $D^{\prime}$ be the space of all distributions on $D$.

Definition 1. Let $\alpha_{r}$ be in $C$ for $r=0,1, \ldots$.

We say that

$$
\alpha=\left[\alpha_{0}, \alpha_{1}, \ldots\right]
$$

is a number vector (2). We denote the vector space of all number vectors, with the usual definition of the sum and product by a scalar, by $\underset{\sim}{\mathrm{C}}$.

$D$ e i i it i i

2. Let $h_{r}$ be in $D^{\prime}$ for $r=0,1, \ldots$.

We say that

$$
\underset{\sim}{\mathrm{h}}=\left[\mathrm{h}_{0}, \mathrm{~h}_{1}, \ldots, \mathrm{h}_{\mathrm{r}}, \ldots\right]
$$

is a distribution veotor. 
If $h_{r+1}=0$ for $i=1,2, \ldots$, we write

$$
\underset{\sim}{h}=\left[h_{0}, h_{1}, \ldots, h_{r}, 0,0, \ldots\right]=\left[h_{0}, h_{1}, \ldots, h_{r}\right]
$$

and if $h_{1}=0$ for $1=1,2, \ldots$ wo write

$$
\underset{\sim}{h}=\left[h_{0}\right]=h_{0} \text {. }
$$

We denote the vector apace of all distribution vectors, with the usual definition of sum and product by a scalar, by $\underset{\sim}{D}$.

$D_{\theta} \pm 1 n i t i 0 n$ 3. Let $\underset{\sim}{g}=\left[\mathrm{h}_{0}, \mathrm{~h}_{1}, \ldots, \mathrm{h}_{\mathrm{r}}, \ldots\right]$ bo in $D_{\sim}^{\prime}$ and let $\varphi$ be in $D$. We define $\underset{\sim}{(h, \varphi)}$ to be the number vector

$$
(\underset{\sim}{h}, \varphi)=\left[\left(h_{0}, \varphi\right),\left(h_{1}, \varphi\right), \ldots,\left(h_{r}, \varphi\right), \ldots\right] .
$$

Def 1 a 1 t 1 on 4. Let $\underset{\sim}{h}=\left[h_{0}, h_{1}, \ldots, h_{r}, \ldots\right]$ be in $\underset{\sim}{D^{\prime}}$. We define the derivative $\underset{\sim}{h^{\prime}}$ of $\underset{\sim}{h}$ by

$$
\stackrel{h^{\prime}}{\sim}=\left[h_{0}^{\prime}, h_{1}^{\prime}, \ldots, h_{1}^{\prime}, \ldots\right] \text {. }
$$

I $h \in \circ \mathrm{r}$ a 1 . Let $\underset{\sim}{\mathrm{h}}=\left[\mathrm{h}_{0}, h_{1}, \ldots, h_{r}, \ldots\right]$ be in $D^{\prime}$ and let $\varphi$ be in $D$. Then

$$
(\underset{\sim}{\mathrm{h}}, \varphi\rangle=-\left(\frac{\mathrm{h}}{\sim}, \varphi^{\prime}\right) .
$$

The proof of the theorem follow日 easily.

D $11 \mathrm{n} \pm \mathrm{t} i \mathrm{n}$ 5. Let $\rho$ be a fixed function in D having the properties:

$$
\rho(x)=0 \text { for }|x| \leqslant 1 \text {, }
$$$$
p(x) \geqslant 0 \text {. }
$$

$$
\rho(x)=\rho(-x) \text {, }
$$$$
\int_{-1}^{1} \rho(x) d x=1
$$

Wo define the eunction $\delta_{\nu}$ by $\delta_{\nu}(x)=\nu \rho(\nu x)$ for all $\nu>0$.
De $f$ infition 6. Iat $\rho$ and $g$ bo in $D^{\prime}$ and lot $B_{\nu}=g * \delta_{\nu}$. If there exist $h_{0}, h_{1}, \ldots, h_{r}$ in $D^{\prime}$ euch that

$$
(f, g, \varphi)=\sum_{1=0}^{r}\left(h_{1}, \varphi\right) \nu^{1}+\Delta(\nu) \text {. }
$$

for arbitrary $\varphi$ in $D$, where

$$
\lim _{\nu \rightarrow \infty} \Delta(\nu)=0
$$

( $\Delta$ could depend on $\varphi$ as well), we define the product $\rho \circ g$ 1n $D^{\prime}$ by

$$
f \circ g=\left[h_{0}, h_{1}, \ldots, h_{x}\right] \text {. }
$$

We say that $h_{0}$ ts the fintte part of $\rho \circ \mathrm{g}$. If $h_{i} \neq 0$ for some $1 \geqslant 1$, we write

$$
p \cdot f \cdot(f \circ g)=h_{0}
$$

and if $h_{1}=0$ for $i=1,2, \ldots, r$, we write

$$
f \circ g=h_{0} \text {. }
$$

The orem 2. The product mol is a generalization of the uaual product in $D^{\prime}$ when one of the distributions 19 a smooth function, 1.e, $f \circ g=f . g$ for all $f \in D^{\prime}$ and all $\mathrm{g} \in \mathrm{C}^{\infty}$.

The above theorem is just an interpretation of Definition 6 , having in mind as well that $g_{p} \varphi \rightarrow B \varphi$ in the test-topology of $D$ when $g$ is a smooth function and $\varphi$ 1s in $D$.

$T h e \circ r \cdot m$ 3. Let $f$ and $g$ be in $D^{\prime}$ and suppose that the produots $f^{\prime} \circ g$ (or $f \circ g^{\prime}$ ) and $f \circ g$ are $1 n D^{\prime} \cdot$ Then the product $f \circ g^{\prime}$ (or $f^{\prime} \circ g$ ) is in $\underset{\sim}{D^{\prime}}$ and

$$
(f \circ g)^{\prime}=f^{\prime} \circ g+f \circ g^{\prime} \text {. }
$$


P r $\circ \circ f$. Suppose

$$
\begin{aligned}
& \left(f, g_{\nu} \varphi\right)=\sum_{i=0}^{r}\left(h_{i}, \varphi\right) \nu^{i}+\Delta(\nu), \\
& \left(f^{\prime}, g_{\nu} \varphi\right)=\sum_{i=0}^{r}\left(k_{1}, \varphi\right) \nu^{i}+\Delta_{1}(\nu),
\end{aligned}
$$

for arbitrary $\varphi$ in $D$, so that

$$
\begin{aligned}
& f \circ g=\left[h_{0}, h_{1}, \ldots, h_{r}\right], \\
& f^{\prime} \circ g=\left[k_{0}, k_{1}, \ldots, k_{r}\right] .
\end{aligned}
$$

Then

$$
\left(\left(f_{g_{\nu}}\right)^{\prime}, \varphi\right)=-\left(f_{g_{\nu}}, \varphi^{\prime}\right)=\left\langle f_{B_{\nu}^{\prime}}^{\prime}+f^{\prime} g_{\nu}, \varphi\right)
$$

and so

$$
\begin{aligned}
\left(f, g_{\nu}^{\prime} \varphi\right) & =-\left(f, g_{\nu} \varphi^{\prime}\right)-\left(f^{\prime}, g_{\nu} \varphi\right)= \\
& =-\sum_{i=0}^{r}\left(h_{i}, \varphi^{\prime}\right) \nu^{i}-\Delta_{2}(\nu)-\sum_{i=0}^{r}\left(h_{1}, \varphi\right) \nu^{i}-\Delta_{1}(\nu) .= \\
& =\sum_{i=0}^{r}\left(h_{1}^{\prime}-k_{i}, \varphi\right) \nu^{1}-\left(\Delta_{1}+\Delta_{2}\right)(\nu)
\end{aligned}
$$

for some function $\Delta_{2}$, where

$$
\lim _{\nu \rightarrow \infty} \Delta_{2}(\nu)=\lim _{\nu \rightarrow \infty}\left(\Delta_{1}+\Delta_{2}\right)(\nu)=0
$$

It follows that the product $f^{\circ} \circ \mathrm{B}^{\prime}$ is $1 \mathrm{n} \underset{\sim}{D^{\prime}}$ and

$$
f \circ g^{\prime}=\left[h_{0}^{\prime}-k_{0}, h_{1}^{\prime}-k_{1}, \ldots, h_{r}^{\prime}-k_{r}\right]=(f \circ g)^{\prime}-f^{\prime} \circ g .
$$

Tha results of the theorem follows.

$$
-404-
$$

We now put for simplicity

$$
\rho_{1}=\rho^{(i)}(0)
$$

for $i=0,1, \ldots$ so that in perticular

$$
\rho_{i}=0
$$

for odd $i$.

$$
\begin{aligned}
& \text { Theorem 4. Tha product } \delta(p) \circ \delta^{(q)} \text { is in } D^{\prime} \text { and } \\
& \delta^{(p)} \circ \delta^{(q)}=h(p, q)=\left[h_{0}(p, q), h_{1}(p, q), \ldots, h_{p+q}(p, q)\right]
\end{aligned}
$$

for $p, q=0,1,2, \ldots$, where

$h_{i}(p, q)= \begin{cases}0, & 0 \leqslant 1 \leqslant q, \\ (-1)^{i-q-1}\left({ }_{i-q-1}^{p}\right) \rho_{i-1} \delta^{(p+q+1-i)}, & q<1 \leqslant p+q+1,\end{cases}$

and $(\underset{q}{p})$ denotes the binomial coefficient

$$
\left(\begin{array}{l}
p \\
q
\end{array}\right)=\frac{p !}{q !(p-q) !}
$$

In particular

$$
\begin{aligned}
& \delta^{2}=\delta \circ \delta=\left[0, \rho_{0} \delta\right], \\
& \delta^{\prime} \circ \delta=\left[0, \rho_{0} \delta^{\prime}\right], \\
& \delta \circ \delta^{\prime}=0 .
\end{aligned}
$$

So, we ase that the multiplication operation "o" 18 a nonmodemative operation.

The $\mathrm{F}$. $\mathrm{m}$ 5. The producte $x_{+}^{p} \circ \delta^{(q)}$ and $\delta^{(q)} \circ x_{+}^{p}$ are in $D^{\prime}$ and

$$
x_{+}^{p} \circ 5^{(q)}=\underset{\sim}{b}(p, q)=\left[h_{0}(p, q), h_{1}(p, q), \ldots, h_{q-p}(p, q)\right]
$$

for $p=0,1, \ldots, q$ and $q=0,1,2, \ldots$, where

- 405 - 
$h_{1}(p, q)=\left\{\begin{array}{l}\frac{1}{2}(-1)^{p}\left(\frac{q}{p}\right) \text { p! } \delta^{(q-p)}, \quad 1=0, \\ (-1)^{p-1}\left(\frac{q-i}{p}\right) \text { pl } \rho_{1-1} \delta^{(q-p-1)}, \quad 1 \leqslant 1 \leqslant q-p\end{array}\right.$

and

$$
\sigma^{(q)} \circ x_{+}^{p}=\underset{\sim}{k}(p, q)=\left[k_{0}(p, q), k_{1}(p, q), \ldots, k_{q \sim p}(p, q)\right]
$$

for $p=0,1, \ldots, q$ and $q=0,1, \ldots$, where

$k_{1}(p, q)=\left\{\begin{array}{l}\frac{1}{2}(-1)^{p}\left(\frac{q}{p}\right) p ! \delta^{(q-p)}, \quad 1=0 \\ (-1)^{p-1}\left(\frac{q}{p+1}\right) p ! \rho_{1-1} \delta^{(q-p-1)}, 1 \leqslant 1 \leqslant q-p .\end{array}\right.$

In particuiar

$$
x_{+}^{p} \circ \delta^{(p)}=\delta^{(p)} \circ x_{+}^{p}=\frac{1}{2}(-1)^{p} p ! 5
$$

for $p=0,1,2, \ldots$.

These theoreme are equivalent to Theorem 3 and 4 proved in $[1]$.

We now consider the product of two distribution vectora. For convenience we note that $D^{\prime}$ is 18omorphio to the epaoe of power gerieg in an indeterminate.. $v$ having distributions a coefficientis. Under thia netural isomorphiem we write

$$
f=\left[f_{0}, f_{1}, \ldots, f_{r}, \ldots\right]=\sum_{r=0}^{\infty} e_{r} \nu^{\nu^{r}} .
$$

Def 1 n 1 t $10 \mathrm{n}$ 7. Lat

and

$$
\stackrel{f}{\mathcal{f}}=\left[f_{0}, f_{1}, \ldots, f_{r} \ldots .\right] \text { L } \sum_{r=0}^{\infty} f_{r} \nu^{r}
$$

$$
\underset{\sim}{g}=\left[8_{0}, 8_{1}, \ldots, 8_{x}, \ldots\right] \equiv \sum_{r=0}^{\infty} 8_{x} \nu^{r}
$$

- 406 - be in $D^{\prime}$ and suppose thet $P_{r}{ }^{\circ} B_{g}$ exist for all $r, \theta=0,1, \ldots$ (in the sense of Definition 6 ) and

$$
f_{r}=g_{8} \sum_{m=0}^{ل j s} h_{r e m} p^{m}
$$

for $r, s=0,1, \ldots$ for some distributiond $h_{r s m}$ and some integers $\mu_{r g} \geqslant 0$. Let now put

$$
h_{n}=\sum_{r=0}^{\infty} \sum_{g=0}^{\infty} \sum_{m=0}^{H_{r s}} h_{r \text { am }} \quad(r+s+m=n)
$$

for $\mathrm{n}=0,1,2, \ldots$. We define the product $\underset{\sim}{f} \circ \mathrm{g}$ in $\mathrm{N}^{\prime}$ by

$$
\begin{aligned}
& \underset{\sim}{f} \circ g\left(\sum_{r=0}^{\infty} f_{r} \nu^{r}\right) \circ\left(\sum_{g=0}^{\infty} g_{g} \nu^{g}\right)=\sum_{r=0}^{\infty} \sum_{g=0}^{\infty}\left(f_{r} \circ g_{g}\right) \nu^{r+s}= \\
& \sum_{r=0}^{\infty} \sum_{s=0}^{\infty}\left(\sum_{m=0}^{\mu r s} h_{r s m^{\nu^{m}}}\right) \nu^{r^{2}+8}= \\
& =\sum_{n=0}^{\infty} h_{n}{ }^{\nu}{ }^{n} \equiv\left[h_{0}, h_{1}, \ldots, h_{n}, \ldots\right]
\end{aligned}
$$

and say that $h_{0}$ is the finite part of $\underset{\sim}{f} \circ$.

Ih o o s m 6. Let $\underset{\sim}{f}$ and $g$ be 1 in $D^{\prime}$ and suppose that the produots $\underset{\sim}{f} \circ g$ and $\underset{\sim}{f^{\prime}} \circ g\left(\right.$ or $\left.\underset{\sim}{f} \circ g^{\prime}\right)$ are $1 n \underset{\sim}{D^{\prime}}$. Then the produot $\underset{\sim}{f} \circ{\underset{\sim}{g}}^{\prime \prime}$ (or $\underset{\sim}{f^{\prime} \circ g}$ ) 10 in $\underset{\sim}{D^{\prime}}$ and

$$
(\underset{\sim}{f} \circ g)^{\prime}=\underset{\sim}{f^{\prime}} \circ g+\underset{\sim}{f} \circ g^{\prime}
$$

P r o of. Suppose

$$
\underset{\sim}{f} \equiv \sum_{r=0}^{\infty} f_{r} \nu^{r}, \quad \underset{\sim}{g} \equiv \sum_{r=0}^{\infty} B_{r} \nu^{r} .
$$


Then

and

$$
\underset{\sim}{f} \stackrel{g}{g} \equiv \sum_{r=0}^{\infty} \sum_{s=0}^{\infty}\left(f_{r} \circ B_{s}\right) \nu^{r+s}
$$

$$
\underset{\sim}{f^{\prime} \circ g}=\sum_{r=0}^{\infty} \sum_{s=0}^{\infty}\left(f_{r}^{\prime} \circ 8_{g}\right)^{r+s}
$$

Bo that the groducte $f_{r} \circ B_{g}$ and $f_{r}^{\prime} \circ g_{g}$ are in $D_{N}^{\prime}$. By Theorem 3 the product $f_{r} \circ g_{G}^{\prime}$ is in $D^{\prime}$ and

Thus

$$
\left(f_{r} \circ g_{r}\right)^{\prime}=f_{r}^{\prime} \circ B_{g}+f_{r} \circ g_{s}^{\prime} .
$$

$$
\begin{aligned}
& (j \circ g)^{\prime} \equiv \sum_{r=0}^{\infty} \sum_{n=0}^{\infty}\left(f_{n} \circ a_{a}\right)^{\prime} r+8= \\
& =\sum_{r=0}^{\infty)} \sum_{g=0}^{\infty}\left(f_{r}^{\prime} \circ g_{g}+f_{r} \circ g_{E}^{\prime}\right) \nu^{r+B}
\end{aligned}
$$

which implies the extotunce of $\underset{\sim}{\mathrm{f}}{ }^{\circ}{\underset{\sim}{\prime}}^{\prime}$ and

$$
(\underset{\sim}{f} \circ g)^{\prime}=\underset{\sim}{f^{\prime}} \circ \underset{\sim}{g}+\underset{\sim}{f} \circ \underset{\sim}{g} \cdot
$$

Bxampla 1. $(\delta, \delta, \varphi)=(\delta, \nu \rho(\nu x) \varphi(x))=\nu \rho(0) \varphi(0)=$ $=\nu \rho_{0}(\delta, \varphi)$ for arbitrary $\varphi$ in $D$ and so

$$
\delta^{2} \equiv \delta \circ \delta=\left[0, \rho_{0} \delta\right] \text {. }
$$

Exsmplo 2. $\delta^{3} \equiv \delta \circ \delta \circ \delta=\nu^{2} \rho_{0}^{2} \delta=\left[0,0, \rho_{0}^{2} 5\right]$. Wore general for the n-th power of the delta-funotion $\delta$ we
obtain

$$
\delta^{n}=\nu^{n-1} \rho_{0}^{n-1} \delta=\left[0,0, \ldots, \rho_{0}^{n-1} \delta\right] .
$$

Bxample 3. $\left(\delta, \delta,(\varphi)=\left\langle\delta, \nu^{2} \varphi^{\prime}(\nu x) \varphi(x)\right)=\right.$

$=\nu^{2} \rho^{\prime}(0) \varphi(0)=0$ for arbitrary $\varphi$ in $D$ and so

$$
\delta \circ \delta^{\prime}=0
$$

B $\times$ a p 1 e 4. Using Theorem 3 we see that $\delta$ to 1a in $\underset{\sim}{D}$ and

$$
\delta^{\prime} \circ \delta=(\delta \circ \delta)^{\prime}-\delta \circ \delta^{\prime}=\left[0, \rho_{0} \delta^{\prime}\right] \text {. }
$$

Ex a mplo, 5. $\left(\delta^{\prime}, \delta_{\gamma}^{\prime} \varphi\right)=\left(\delta^{\prime}, \nu^{2} \rho^{\prime}(\nu x) \varphi(x)\right)=$ $=-\nu^{3} \rho^{\prime \prime}(0) \varphi(0)+\nu^{2} \rho^{\prime}(0) \varphi^{\prime}(0)=-\nu^{3} \rho^{\prime \prime}(0)(\delta, \varphi)$ for arbitrary $\varphi$ in $D$ and 80

$$
\delta^{\prime} \circ \delta^{\prime}=\left[0,0,0,-\rho_{2} \delta\right] .
$$

These four results are, of course, particular cases of Theorem 4.

Ex a x p $1 \circ$ 6. $\left[\delta, \delta^{\prime}\right] \circ\left[\delta^{\prime} ; 5\right] \equiv\left(\delta+\nu \delta^{\prime}\right) \circ\left(\delta^{\prime}+, \delta\right)=$ $=\delta \circ \delta^{\prime}+\nu \delta \circ \delta+\nu \delta^{\prime} \circ \delta^{\prime}+\nu^{2} \delta^{\prime} \circ \delta=\left[0,0, \rho_{0} \sigma, \rho_{0} \delta^{\prime},-\rho_{2} \delta\right.$.

We finsily congider integration in $D^{\prime}$ and $D^{\prime}$.

De $f$ I 1 t $10 \mathrm{n}$ 8. Let $\rho$ be in $D^{\prime}$, let $\mu$ be a measure in $R$ and let 6 bo a measureblo subset of $R$. Te saj that $f$ is integrable on $\sigma$ if there exiate an integer $m \geqslant 0$ and complex coeffiolente $\alpha_{0}, \alpha_{1}, \ldots, \alpha_{m}$ for whioh

$$
\int_{\sigma} f_{\nu}(x) d \nu(x)=\sum_{i=0}^{m} \alpha_{1} \nu^{1}+\Delta(\nu)
$$

where $f_{\nu}=\rho * \delta_{\nu}$ and

$$
\lim _{\nu \rightarrow \infty} \Delta(\nu)=0
$$

We then write

$$
\int_{\sigma} f(x) d \mu(x)=\left[\alpha_{0}, \alpha_{1}, \ldots, \alpha_{m}\right] \equiv \sum_{1=0}^{m} \alpha_{1} \nu^{1}
$$

and say that $\alpha_{0}$ is the fintte part of the integral. 


$$
\begin{aligned}
& \text { Example 7. } \int_{-\infty}^{\infty} \delta(x) d x=1 \text {. } \\
& \text { Example 8. } \int_{0}^{\infty} \delta_{\nu}(x) d x=\int_{0}^{1} p \rho(\nu x) d x=\frac{1}{2} \text { and } \theta 0 \\
& \int_{0}^{1} \delta(x) d x=\frac{1}{2} \text {. } \\
& \text { Ex a d p } 1 \text { e 9. } \int_{-\infty}^{\infty} \delta^{\prime}(x) d x=0 \text {. } \\
& \text { Example 10. } \int_{0}^{1} \delta_{\nu}^{\prime}(x) \mathrm{d} x=\int_{0}^{1} j^{2} \rho^{\prime}(\nu x) \mathrm{d} x=-\nu \rho
\end{aligned}
$$

and so $\int_{0}^{1} \delta^{\prime}(x) d x=\left[0,-\rho_{0}\right]$.

The orem 7. For all $f \in D^{\prime}$ and all $\varphi \in D$ we have:

$$
\int_{-\infty}^{\infty}(f \circ \varphi) d x \equiv \int_{-\infty}^{\infty} f(x) \varphi(x)=(f, \varphi)
$$

F $r \circ \circ f$. It is well known that $(f \varphi\rangle_{\nu} \underset{\nu \rightarrow \infty}{f} \varphi$ in tho topology of $\varepsilon^{\prime}\left(\varepsilon^{\prime}\right.$ is the space of all distributions with compact supports) so that

$$
\lim _{\nu \rightarrow \infty} \int_{-\infty}^{\infty}(f \varphi)_{\nu} d x=(f \varphi, 1)=(f, \varphi) .
$$

The proof is finished.

$$
\begin{aligned}
& D \in f i n i t i 0 n \text {. Let } \\
& \qquad \stackrel{f}{\sim}\left[f_{0}, f_{1}, \ldots, f_{r}, \ldots\right] \equiv \sum_{r=0}^{\infty} f_{r} \nu^{r}
\end{aligned}
$$

be in $\underset{\sim}{D^{\prime}}$ and suppose that $f_{p}$ is integrable on 6 with

$$
\int_{\delta} f_{x}(\dot{x}) d \mu(x) \equiv \sum_{i=0}^{m_{x}} \alpha_{r i 1^{2}} \pm
$$

for $r=0,1, \ldots$. We sey that $\underset{\sim}{f}$ is integrable on $\sigma$ and write

$$
\int_{\sigma} f(x) d \mu(x) \equiv \sum_{r=0}^{\infty} \sum_{i=0}^{m_{r}} d r_{1} \nu^{r+i} \equiv\left[d_{0}, d_{1}, \ldots, d_{n}, \ldots\right]
$$

where

$$
d_{n}=\sum_{r=0}^{\infty} \sum_{i=0}^{m_{r}} d r_{i}, \quad(r+i=n)
$$

for $n=0,1, \ldots$ and say that $d_{0}$ is the finite part of the integral.

$$
\text { Example 11. } \int_{0}^{1}\left[\delta(x), \delta^{\prime}(x)\right] d x=\left[1 / 2,0,-\rho_{0}\right] \text {. }
$$

We see that the integral of a given distribution vector (if exista) is a number vector.

$R$ a a $r k$. The reader could romein disappointed at the fact that the multiplication operation introduced in our paper is nonssoociative which follows directly from the examplo

$$
\delta^{\prime} \circ\left(\delta^{\circ} \circ \delta^{\prime}\right) \neq\left(\delta^{\prime} \circ \delta^{\prime}\right) \circ \delta^{\prime}
$$

Recall, however, that according to the well-known interpretetion of the Schwartz example

$$
\left(x^{-1} \cdot x\right) 5(x) \neq x^{-1}(x \cdot 8(x))
$$

It is principally impossible to supply the distribution space or any of 1ts enlargements (in partioular, the space of di-

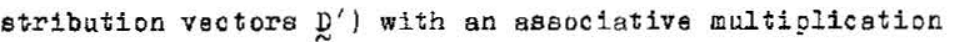
operstion.

\section{REFERENCES}

[1] B. Fis her: Products of distributions defined by a vector, Proceading of the Third International Conference on Complex Analjais and Applications, Varna (1985), (to appoar). 
[2] Chr. Ya. Chr 1 s tov, T.D. Tod O $\mathrm{C} \circ \mathrm{V}:$ Asymptotio numbers - algebraio operations with them, Serdica, Bulgaricae Mathematicae Publicationes, v01.2 (1976) 87-102.

DEPARTMENT OF MATHEMATICS, THE UNIVBRSITY, LEICESTER LEI TRH, U.K.:

BULGARIAN ACADEMY OF SCIENCES, INSTITUTE OF NUCLEAR RESEARCH AND NUCLBAR ENERGY, 1784 SOPIA, BULGARIA

Received October 10, 1985. 